Infinite Dimensional Analysis, Quantum Probability

and Related Topics

Vol. 18, No. 4 (2015) 1599001 (2 pages)

(C) World Scientific Publishing Company

DOI: $10.1142 / \mathrm{S} 0219025715990015$

\title{
Author index (Vol. 18)
}

Accardi, L., Ji, U. C. and Saitô, K., Higher order multidimensional extensions of Cesàro theorem

Alpay, D., Jorgensen, P. E. T. and Kimsey, D. P., Moment problems in an infinite number of variables

Applebaum, D. and van Neerven, J., Second quantisation for skew convolution products of infinitely divisible measures

Bell, D., The Malliavin calculus and hypoelliptic differential operators

Ben Ammou, B. K. and Lanconelli, A., On stochastic differential equations driven by the renormalized square of the Gaussian white noise

Bock, W. and Grothaus, M., The Hamiltonian path integrand for the charged particle in a constant magnetic field as white noise distribution

Bolaños-Servin, J. R. and Quezada, R., The $\Theta$-KMS adjoint and time reversed quantum Markov semigroups

Bolaños-Servin, J. R. and Fagnola, F., On the range of the generator of a quantum Markov semigroup
Bożejko, M. and Bożejko, W., Generalized Gaussian processes and relations with random matrices and positive definite functions on permutation groups

Bożejko, W., see Bożejko

Carbone, R. and Martinelli, A., Logarithmic Sobolev inequalities in non-commutative algebras

Cordoni, F. and Di Persio, L., Invariant measure for the Vasicek interest rate model in the Heath-JarrowMorton-Musiela framework

Datt, G. and Porwal, D. K., On commutativity of weighted Hankel operators and their spectra

18 (2015) 1550025

Demni, N. and Mouayn, Z., Analysis of generalized poisson distributions associated with higher Landau levels

Di Persio, L., see Cordoni

Fagnola, F., see BolañosServin

18 (2015) 1550010

Fernando, B. P. W. and Sritharan, S. S. Stochastic quasi-linear partial differential equations of evolution

18 (2015) 1550016

García, J. C. and Guerrero-Poblete, F., Some non-equilibrium invariant states for the asymmetric exclusion QMS at level one
18 (2015) 1550020

18 (2015) 1550020

18 (2015) 1550011

18 (2015) 1550022

18 (2015) 1550018

18 (2015) 1550028

18 (2015) 1550022

18 (2015) 1550027

18 (2015) 1550021

18 (2015) 1550007 
Gerhold, M. and Lachs, S., Classification and GNS-construction for general universal products

Grothaus, M., see Bock

Guerrero-Poblete, F., see García

Hamdi, T., Monotone and boolean unitary Brownian motions

Haydarov, F. H., see Rozikov

Holmes, I. and Sengupta, A. N., The Gaussian Radon transform and machine learning

Ji, U. C., see Accardi

Jorgensen, P. E. T., see Alpay

Kalinichenko, A. A., Feynman approximation to integrals with respect to Brownian sheet on Lie groups

Khrennikov, A., Kozyrev, S. and Månsson, A., Hierarchical model of the actomyosin molecular motor based on ultrametric diffusion with drift

Kimsey, D. P., see Alpay

Ko, C. K., Park, S. D. and Yoo, H. J., Glauber dynamics on the cycles: Spectral distribution of the generator

Kozyrev, S., see Khrennikov

Krystek, A. D. and Wojakowski, Ł. J., Conditionally free semistable distributions

Lachs, S., see Gerhold

Lanconelli, A., see Ben Ammou

Liebscher, V., The relation of spatial and tensor product of Arveson systems - the random set point of view

Månsson, A., see Khrennikov

Martinelli, A., see Carbone

Mouayn, Z., see Demni

Newton, N. J., Information geometric nonlinear filtering

Park, S. D., see Ko

Parthasarathy, K. R. and Sengupta, R., From particle counting to Gaussian tomography

18 (2015) 1550004 18 (2015) 1550010

18 (2015) 1550007

18 (2015) 1550012

18 (2015) 1550006

18 (2015) 1550019

18 (2015) 1550030

18 (2015) 1550024

18 (2015) 1550008

18 (2015) 1550013

18 (2015) 1550024

18 (2015) 1550002

18 (2015) 1550013

18 (2015) 1550015

18 (2015) 1550004

18 (2015) 1550025

18 (2015) 1550029

18 (2015) 1550013

18 (2015) 1550011

18 (2015) 1550028

18 (2015) 1550014
Porwal, D. K., see Datt

Pronk, M. and Veraar, M., Forward integration, convergence and non-adapted pointwise multipliers

Quezada, R., see BolañosServin, J. R.

Röckner, M. and Trutnau, G., About the infinite dimensional skew and obliquely reflected Ornstein-Uhlenbeck process

Rozikov, U. A. and Haydarov, F. H., Periodic Gibbs measures for models with uncountable set of spin values on a Cayley tree

Saitô, K., see Accardi

Savov, M. and Wang, S.D., Fluctuation limits of a locally regulated population and generalized Langevin equations

Sengupta, A. N., see Holmes

Sengupta, R., see Parthasarathy

Sritharan, S. S., see Fernando

Trutnau, G., see Röckner

Ulrich, M., Construction of a free Lévy process as high-dimensional limit of a Brownian motion on the unitary group

van Neerven, J., see Applebaum

Veraar, M., see Pronk

Wang, F.-Y. and Zhang, X., Degenerate SDEs in Hilbert spaces with rough drifts

Wojakowski, Ł. J., see Krystek

Yoo, H. J., see Ko

Zhang, X., see Wang
18 (2015) 1550026

18 (2015) 1550009

18 (2015) 1550002

18 (2015) 1550023

18 (2015) 1550018

18 (2015) 1550005

18 (2015) 1550016

18 (2015) 1550031

18 (2015) 1550006

18 (2015) 1550030

18 (2015) 1550009

$18(2015) 1550019$

18 (2015) 1550023

18 (2015) 1550021

18 (2015) 1550031

18 (2015) 1550018

18 (2015) 1550003

18 (2015) 1550005

18 (2015) 1550015

18 (2015) 1550002

18 (2015) 1550026 